\title{
Real-time Ultrasound Volume Navigation Guided Transforaminal Percutaneous Endoscopic Lumbar Discectomy in Anatomic Variation: A Case Report
}

\author{
Yingying Li, MM ${ }^{a}$, Peng Huang, MD ${ }^{b}$, Shoupeng Li, MM ${ }^{a}$, Mingbo Zhang, MD ${ }^{a, *}$ \\ ${ }^{a}$ Department of Ultrasound, First Medical Center of Chinese PLA General Hospital, Beijing, China; ${ }^{b}$ Department of Orthopedics, First \\ Medical Center of Chinese PLA General Hospital, Beijing, China \\ Received October 16, 2019; revision received November 14; accepted November 28.
}

\begin{abstract}
Percutaneous endoscopic lumbar discectomy (PELD) has become a mature and mainstream minimal invasive surgical technique in the treatment of lumbar disc herniation (LDH). During the operation, transforaminal puncture is considered as a critical and complicated step which is usually carried out under the guidance of X-ray. However, radiation exposure brings a potential threat to the health of the patients and the surgeons. Besides, nerve roots and vessels may be injured since they are invisible on X-ray. So we still need a real-time accurate image guiding system, especially in difficult cases with anatomic variation. Here we report a case to describe a new method, volume navigation with fusion of real-time ultrasound and CT images, to guide PELD in a patient with right L4-L5 LDH who had anatomic variation of lumbar sacralization. Ultrasound volume navigation guided puncture and cannulation process lasted only 10 minutes and the operation decompression time lasted 60 minutes. The total emission radiation dose was 9mGy. The straight leg elevation increased from 50 to 80 degrees after PELD. The Visual Analog Scale (VAS) of low back pain reduced from 5 to 1 and leg pain reduced from 7 to 1 immediately after PELD. This is the first case of ultrasound volume navigation in guidance of the postural lateral transforaminal puncture and cannulation process of PELD.
\end{abstract}

Key words: Ultrasound; Volume navigation; CT; Transforaminal percutaneous endoscopic lumbar discectomy; Anatomic variation

Advanced Ultrasound in Diagnosis and Therapy 2019;04:193-196

DOI: 10.37015/AUDT.2019.191232

Percutaneous endoscopic lumbar discectomy (PELD) has become a mature and mainstream minimal invasive surgical technique in the treatment of lumbar disc herniation $(\mathrm{LDH})[1,2]$ and the transforaminal puncture is considered as a critical and complicated step which is usually carried out under the guidance of X-ray. However, radiation exposure brings a potential threat to the health of the patients and the surgeons. Besides, nerve roots and vessels may be injured because they are invisible on X-ray. So we still need a real-time accurate image guiding system, especially in difficult cases with anatomic variation. In our study, we explored a novel ultrasound (US) volume navigation (V Nav) technique to guide transforaminal PELD and we reported its application in a patient with anatomic variation of lumbar sacralization.

\section{Case report}

A 39-year-old male with low back pain, right leg pain and numb for more than 6 months came to our hospital. Physical examination showed bilateral L5-S1 vertebral spinous process tenderness and percussion pain without radiation. The right straight leg rising and strengthen exam was positive (50 degrees). MRI showed the patient had an anatomic variation that the fifth lumber vertebra

* Corresponding Author: Department of Ultrasound, First Medical Center of Chinese PLA General Hospital, 28 Fuxing Road, Beijing, China.

e-mail:owsifanduizhe@126.com 
was sacralizaed which suggested the actual lesion was in L4-5 level. The patient was diagnosed as LDH (right margin type) and needed PELD for decompression. US $\mathrm{V}$ Nav was used to guide the puncture and cannulation process. Institutional approval for the procedure was obtained by the ethics committee of our hospital in accordance with the tenets of the Declaration of Helsinki. Written informed consent was obtained from the patient.

The patient lay in a prone position with a pillow under belly. The intersection of the line connecting the highest points of the bilateral iliac crests and the posterior midline was selected as the center, and 5 points were randomly choosen with the center of the surgery field in a circle within a radius of $5 \mathrm{~cm}$. One metal snap was attached to each point as an external marker. A Siemens 64 Slice CT scanner (Siemens Sensation Open, Germany) was used to complete the scan and threedimensional (3D) reconstruction of the lumbar spine in the prone position. The range of the scan was from the superior margin of the L1 vertebral body to the inferior margin of the $\mathrm{S} 2$ vertebral body. The CT scan parameters were as follows: slice thickness $=1.5 \mathrm{~mm}$; interlayer distance $=1.5 \mathrm{~mm}$; speed of rotation $=2 \mathrm{sec} /$ lap; tube voltage $=120 \mathrm{kV}$; current $=150 \mathrm{~mA}$; and matrix $=512 \times 512$. A portable Esaote MyLabTwice Ultrasound System (Esaote, Genoa, Italy) with a C5-1S convex array transducer (central frequency $5 \mathrm{MHz}$, frequency range from $1 \mathrm{MHz}$ to $8 \mathrm{MHz}$ ) and volume navigation system was used in this study. An electromagnetic tracker was placed next to the patient in the prone position. The distance between the US probe and the tracker should be less than $80 \mathrm{~cm}$. Before registration, electromagnetc tracking locators were mounted on the US probe and the tail of the needle, to locate the position of the US probe and the needle tip in magnetic field. The position of the tracker and the five metal snaps remained unchanged during the entire process. The three-dimentional CT data were imported into the US V Nav device, all the 5 points on the body surface were accurately identified and locked one by one. Registration of the inner marker was established after registration of the five outer markers were acquired. The apex of the L4 spinous process was identified on the sagittal plane of the CT image (Fig. 1) and locked as inner marker, meanwhile the corresponding point was identified on the real-time US image and locked (Fig. 2).

After image fusion, the patient was sterilized. One physician with more than 4 years experience of interventional US performed the US V Nav guidance. One spinal surgeon with more than 5 years experience in PELD performed the puncture and cannulation procedure under the guidance. The position of the electromagnetic tracker, the markers and the patient must not be moved during the whole procedure. The intervertebral foramen of right L4-5 on the CT transverse plane was selected as the target, which was displayed and locked by US simultaneously. The US probe was maintained on the target circle plane with the virtual needle path on it. Then the needle was inserted into the target circle, the intervertebral foramen of right L45 , along the line that was calculated by the US V Nav system shown on the screen (Fig. 3). When the puncture needle and the target cirecle were on the same plane, the virtual needle path was highlighted to guide the needle puncture. After the puncture was successfully completed, CT with 3D reconstruction was repeated to verify the needle position (Fig. 4). A guide wire was then inserted through the spinal needle, and the spinal needle was removed. Thereafter, an obturator and reamer were introduced along the guidewire. Finally, the expanders and endoscope outer sheath were inserted through the guidance wire one after another.

US V Nav guided puncture and cannulation process

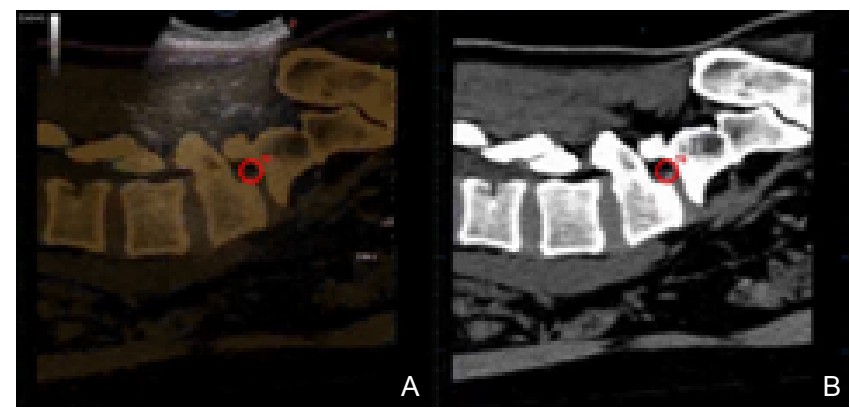

Figure 1 The saggital images of the lumbar spine. (A) Fusion image of ultrasound and CT; (B) CT image. The red circle (T0) showed the puncture target, the intervertebral foramen of right L4-5.

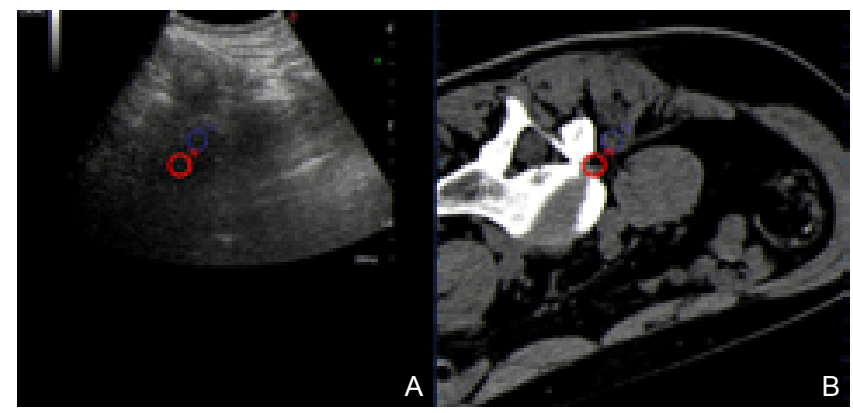

Figure 2 The transverse images of the lumbar spine at the same plane. (A) The target (T0) showed a hypoechoic area on ultrasound image; (B) The intervertebral foramen of the right L4-5 was located on the CT image and was depicted as the red circle target (T0).

of the L4-L5 level lasted 10 minutes. The positions of puncture needle and working cannula were accurate, tested by CT, with total fluoroscopic time of $8.0 \mathrm{~s}$ and total emission radiation dose of $9 \mathrm{mGy}$. The operation decompression time lasted 60 minutes. The straight leg elevation increased from 50 to 80 degrees after PELD. The VAS of low back pain reduced from 5 to 1 and leg 
pain reduced from 7 to 1, immediately after PELD.

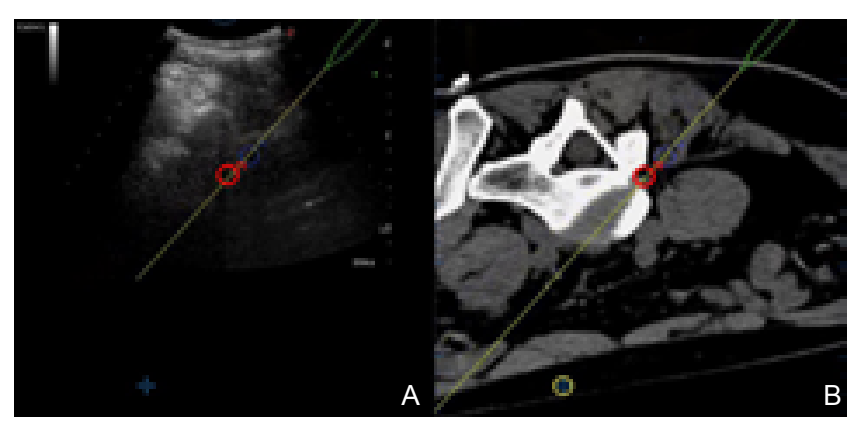

Figure 3 The transverse ultrasound image and CT image of the lumbar spine during puncture process. Yellow Line: puncture path; Green Arrow: needle position. The actural puncture needle was coincided with the virtual needle path and the needle path was highlighted as a yellow line.

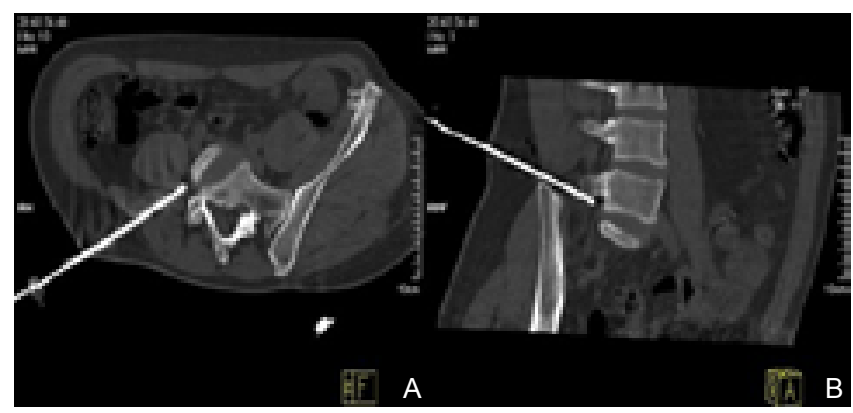

Figure 4 The transverse and saggital CT images of the lumbar spine. The high-density linear image confirmed that the puncture needle was correctly inserted to the intervertebral foramen of right L4-5.

\section{Discussion}

PELD has become a mature and mainstream minimal invasive surgical technique in the treatment of lumbar disc herniation (LDH) [1,2], with the advantages of minimal access trauma, less destruction of the bone and soft tissue, and rapid postoperative recovery. And fluoroscopy is inevitable to ensure the accuracy of puncture and cannulation[3,4]. During the process of PELD, the transforaminal puncture is considered a critical and complicated step by most surgeons and is usually carried out under the guidance of X-ray. However, radiation exposure may increase the incidence of malignant tumor, such as thyroid cancer, skin erythema, leukemia, and so on, especially in difficult patients and surgeons with less experience [5]. In addition, inaccurate puncture may cause injuries of the nerve roots, vessels and the neighboring organs.

US is a convenient, noninvasive, radiation-free, and real-time guiding method, which is widely used in abdominal surgical operations. However, when it comes to lumbar spine, it is hard to display clearly due to complete reflection from the cortical bone. Altough there are some studies of US-guided nerve blocks, US has not been widely used in the guidance of lumbar surgical operation [6-8]. As a newly developed technique, US V Nav with the image fusion technique can visualize realtime dynamic US images and CT or MRI images in the same section simultaneously. It can also diplay the 3D relationship of the virtual needle route and the real needle route on sceen to guide the needle insertion precisely to the target [9-12]. US V Nav has been used in detection and minimally invasive treatment of US invisible liver and breast lesions[13-15]. In our study, we proved that it can also be used in the guidance of PELD operation in LDH patients, especially in cases with anatomic variation. Due to the complexity of the lumbar vertebra and the fixation of bone, we believe US V Nav is also suitable for the navigation of other lumbar minimally invasive operations.

In this case, the young patient was diagnosed as lumbar sacralization by MRI and this anatomic variation made the process of puncture and cannulation more difficult. With the guidance of US V Nav, the needle was advanced to the appropriate targent only one time and this procedure only lasted 10 minutes. The time required for the surgery was largely reduced and the accuracy was improved. Furthermore, while the average fluoroscopy times for a PELD operation used to be 23 , with the total emission radiation dose range of 28.5 30.8 mGy [16], here the radiation dose of US V Nav was significantly reduced to only $9 \mathrm{mGy}$. Future study with larger patient numbers is needed to validate this technique as an alternative to fluoroscopy that improves the safety and accuracy, and decreases the time required for the operation.

In this study, we verified that the US V Nav techenique could overcome the disadvantages of conventional US, display the complicated structures of lumbar vertebras and intervertebral discs, and guide transforaminal puncture precisely with less time and less radiation dose. It can also reduce the damages of surrounding important structures, such as abdominal and retroperitoneal organs, the nerve roots and the large vessels.

In this case, we planned to perform MR images for US V Nav. However, the patient was reluctant to wait for another MR scan. So we used intra-operation CT, which is more convienient. In the future, we may use MRI to confirm the feasibility of replacing the X-ray or CT in PELD.

In conclusion, US V Nav can be used to guide the puncture and cannulation process of PELD, especially in difficult cases with anatomic variation, which can increase the safety and reduce the time duration and radiation dose of the operation. 


\section{Funding}

This study was supported by the clinical research support fund of PLA general hospital (No. 2018XXFC-18).

\section{Conflict of Interest}

The authors have no conflict of interest to declare. The funders had no role in study design, data collection and analysis, decision to publication, or preparation of the paper.

\section{Reference}

[1] Cong L, Zhu Y, Tu G. A meta-analysis of endo-scopic discectomy versus open discectomy for symptomatic lumbar disk herniation. Eur Spine J 2016;25:134-43.

[2] Rasouli MR, Rahimi-Movaghar V, Shokraneh F, Moradi-Lakeh M, Chou R. Minimally invasive discectomy versus microdiscectomy/ open discectomy for symptomatic lumbar disc herniation. Cochrane Database Syst Rev 2014;9:CD010328.

[3] Nellensteijn J, Ostelo R, Bartels R, Peul W, van Royen B, van Tulder M. Transforaminal endoscopic surgery for lumbar stenosis: a systematic review. Eur Spine J 2010;19:879-86.

[4] Birkenmaier C, Komp M, Leu HF, Wegener B, Ruetten S. The current state of endoscopic disc surgery: review of controlled studies comparing full-endoscopic procedures for disc herniations to standard procedures. Pain Physician 2013;16:335-44.

[5] Brenner DJ, Doll R, Goodhead DT, Hall EJ, Land CE, Little JB, et al. Cancer risks attributable to low doses of ionizing radiation: assessing what we really know. Proc Natl Acad Sci U S A 2003;100:13761-6.

[6] Galiano K, Obwegeser AA, Bodner G, Freund M, Maurer H, Kamelger FS, et al. Real-time sonographic imaging for periradicular injections in the lumbar spine: a sonographic anatomic study of a new technique. J Ultrasound Med 2005;24:33-8.

[7] Kim D, Choi D, Kim C, Kim J, Choi Y. Transverse process and needles of medial branch block to facet joint as landmarks for ultrasound-guided selective nerve root block. Clin Orthop Surg
2013;5:44-8.

[8] Chumnanvej S, Wetchagama N, Kounsongtham V. Accuracy of needle-tip localization by ultrasound guidance lumbar selective nerve root block: a prospective clinical study of 78 lumbar nerve roots block. J Med Assoc Thai 2011;94:1451-6.

[9] Wing-Fai Loke, Tae-Young Choi, Maleki T, Papiez L, Ziaie B, Byunghoo Jung. Magnetic tracking system for radiation therapy. IEEE Trans Biomed Circuits Syst 2010;4:223-31.

[10] Jung EM, Friedrich C, Hoffstetter P, Dendl LM, Klebl F, Agha A, et al. Volume navigation with contrast enhanced ultrasound and image fusion for percutaneous interventions: first results. PloS One 2012;7:e33956.

[11] Zacchino M, Almolla J, Canepari E, Merico V, Calliada F. Use of ultrasound-magnetic resonance image fusion to guide sacroiliac joint injections: a preliminary assessment. J Ultrasound 2013;16:111-8.

[12] Sartoris R, Orlandi D, Corazza A, Sconfienza LM, Arcidiacono A, Bernardi SP, et al. In vivo feasibility of real-time MR-US fusion imaging lumbar facet joint injections. J Ultrasound 2017;20:23-31.

[13] Wobser H, Wiest R, Salzberger B, Wohlgemuth WA, Stroszczynski C, Jung EM. Evaluation of treatment response after chemoembolisation (TACE) in hepatocellular carcinoma using real time image fusion of contrast-enhanced ultrasound (CEUS) and computed tomography (CT)--preliminary results. Clin Hemorheol Microcirc 2014;57:191201.

[14] Chang JM, Han W, Moon HG, Yi A, Cho N, Koo HR, et al. Evaluation of tumor extent in breast cancer patients using realtime MR navigated ultrasound: preliminary study. Eur J Radiol 2012;81:3208-15.

[15] Kunishi Y, Numata K, Morimoto M, Okada M, Tanaka K. Efficacy of fusion imaging combining sonography and hepatobiliary phase MRI with Gd-EOB-DTPA to detect small hepatocellular carcinoma. AJR Am J Roentgenol 2012;198:106-14.

[16] Xin G, Shi-Sheng H, Hai-Long Z. Morphometric analysis of the YESS and TESSYS techniques of percutaneous transforaminal endoscopic lumbar discectomy. Clin Anat 2013;26:728-34. 